\title{
Replacement of potatoes with other vegetables and risk of myocardial infarction in the Danish Diet, Cancer and Health cohort
}

Würtz, Anne Mette L.; Hansen, Mette D.; Tjønneland, Anne; Rimm, Eric B.; Schmidt, Erik B.; Overvad, Kim; Jakobsen, Marianne Uhre

Published in:

British Journal of Nutrition

Link to article, DOI:

$10.1017 / \mathrm{S} 0007114521000349$

Publication date:

2021

Document Version

Peer reviewed version

Link back to DTU Orbit

Citation (APA):

Würtz, A. M. L., Hansen, M. D., Tjønneland, A., Rimm, E. B., Schmidt, E. B., Overvad, K., \& Jakobsen, M. U. (2021). Replacement of potatoes with other vegetables and risk of myocardial infarction in the Danish Diet, Cancer and Health cohort. British Journal of Nutrition, 126(11), 1709-1716.

https://doi.org/10.1017/S0007114521000349

\section{General rights}

Copyright and moral rights for the publications made accessible in the public portal are retained by the authors and/or other copyright owners and it is a condition of accessing publications that users recognise and abide by the legal requirements associated with these rights.

- Users may download and print one copy of any publication from the public portal for the purpose of private study or research.

- You may not further distribute the material or use it for any profit-making activity or commercial gain

- You may freely distribute the URL identifying the publication in the public portal 
Replacement of potatoes with other vegetables and risk of myocardial infarction in the Danish Diet, Cancer and Health cohort

Anne Mette L. Würtz* ${ }^{1}$, Mette D. Hansen* ${ }^{1}$, Anne Tjønneland ${ }^{2,3}$, Eric B. Rimm ${ }^{4}$, Erik B. Schmidt $^{5}$, Kim Overvad ${ }^{1,5}$, Marianne U. Jakobsen ${ }^{1,6}$

* Co-first authors

${ }^{1}$ Department of Public Health, Aarhus University, DK-8000 Aarhus C, Denmark

${ }^{2}$ The Danish Cancer Society Research Center, DK-2100 Copenhagen $\varnothing$, Denmark

${ }^{3}$ Department of Public Health, University of Copenhagen, DK-1014 Copenhagen K, Denmark

${ }^{4}$ Channing Division of Network Medicine, Brigham and Women's Hospital, Department of

Medicine, Harvard Medical School, Boston MA, and Departments of Epidemiology and Nutrition, Harvard T.H. Chan School of Public Health, Boston, MA 02115, USA

${ }^{5}$ Department of Cardiology, Aalborg University Hospital, DK-9000 Aalborg, Denmark

${ }^{6}$ Division for Diet, Disease Prevention and Toxicology, National Food Institute, Technical University of Denmark, DK-2800 Kgs. Lyngby, Denmark

\section{Corresponding author:}

Anne Mette Lund Würtz, Department of Public Health, Aarhus University, Bartholins Allé 2, DK8000 Aarhus C, Denmark. E-mail: $\underline{\text { amlw@ph.au.dk }}$

Running title: Vegetables and myocardial infarction.

Key words: cohort study, diet, follow-up study, food replacement, ischemic heart disease, myocardial infarction, potatoes, substitution models, vegetables.

Abbreviations: HR, hazard ratio; ICD, International Classification of Diseases; MI, myocardial infarction.

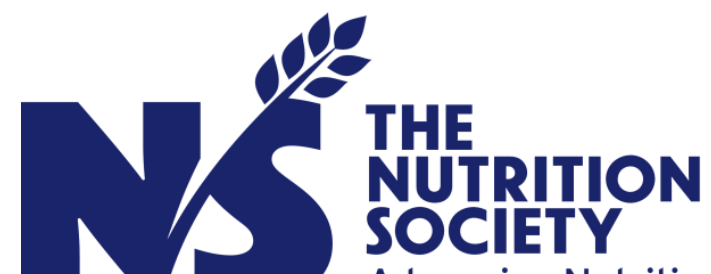

\section{Advancing Nutritional Science}

This peer-reviewed article has been accepted for publication but not yet copyedited or typeset, and so may be subject to change during the production process. The article is considered published and may be cited using its DOI

$10.1017 /$ S0007114521000349

The British Journal of Nutrition is published by Cambridge University Press on behalf of The Nutrition Society 
Accepted manuscript

\begin{abstract}
Intake of vegetables is recommended for the prevention of myocardial infarction (MI). However, vegetables make up a heterogeneous group, and subgroups of vegetables may be differentially associated with MI. The aim of this study was to examine replacement of potatoes with other vegetables or subgroups of other vegetables and the risk of MI. Substitutions between subgroups of other vegetables and risk of MI were also investigated. We followed 29,142 women and 26,029 men aged 50-64 years in the Danish Diet, Cancer and Health cohort. Diet was assessed at baseline by using a detailed validated FFQ. Hazards ratios (HR) with $95 \%$ CI for the incidence of MI were calculated using Cox proportional hazards regression. During 13.6 years of follow-up, 656 female and 1,694 male cases were identified. Among women, the adjusted HR for MI was 1.02 (95\% CI: $0.93,1.13$ ) per $500 \mathrm{~g} /$ week replacement of potatoes with other vegetables. For vegetable subgroups, the HR was $0.93(95 \% \mathrm{CI}: 0.77,1.13)$ for replacement of potatoes with fruiting vegetables and 0.91 (95\% CI: $0.77,1.07)$ for replacement of potatoes with other root vegetables. A higher intake of cabbage replacing other vegetable subgroups was associated with a statistically non-significant higher risk of MI. A similar pattern of associations was found when intake was expressed in $\mathrm{kcal} /$ week. Among men, the pattern of associations was overall found to be similar to that for women. This study supports food-based dietary guidelines recommending to consume a variety of vegetables from all subgroups.
\end{abstract}




\section{INTRODUCTION}

CHD is a leading cause of morbidity and mortality, especially in developed countries ${ }^{(1,2)}$. Prevention of CHD therefore remains a major public health priority. CHD arises from a mixture of genetic and lifestyle factors, and diet is an important modifiable component ${ }^{(3,4)}$. The combined evidence from several prospective cohort studies supports an inverse association between intake of vegetables and risk of $\mathrm{CHD}^{(5,6)}$, and intake of vegetables is recommended by cardiologists and health organizations worldwide for prevention of $\mathrm{CHD}^{(7,8)}$. It is further recommended to eat a variety of vegetables ${ }^{(7)}$. However, studies on the association between subgroups of vegetables and risk of CHD are limited. They have so far shown no associations or inverse associations for intake of especially green leafy vegetables (such as spinach, iceberg, romaine, kale and chard greens) and risk of $\mathrm{CHD}^{(6)}$.

Individuals usually maintain a relatively stable energy intake over time ${ }^{(9)}$, and a higher intake of vegetables is likely to be associated with a lower intake of other energy-providing foods. The replaced food items could be harmful, neutral or beneficial in relation to CHD, and therefore the association between vegetable intake and CHD also depends on the replaced foods in the diet. However, most studies examining the association between vegetable intake and CHD have not specified the replaced foods. Substitution studies comparing specific vegetables with other specified foods are therefore needed to provide detailed knowledge of how different vegetables may affect the risk of $\mathrm{CHD}^{(7,10,11)}$.

Potatoes are classified as a root vegetable from a botanical perspective but are mainly eaten as a staple food like rice or pasta ${ }^{(12)}$. Compared to most other vegetables, potatoes are energy dense, nutrient poor and have a high glycemic index ${ }^{(13,14)}$. Thus, replacement of potatoes with other vegetables may prevent CHD. The aim of our study was to investigate replacement of potatoes with other vegetables or subgroups of other vegetables and risk of myocardial infarction (MI). Substitutions between subgroups of other vegetables and risk of MI were also investigated.

\section{SUBJECTS AND METHODS}

\section{Study population}

The present study is based on data from participants in the Danish Diet, Cancer and Health cohort. From December 1993 through May 1997, 79,729 women and 80,996 men aged 50-64 years were invited by mail to participate in the cohort. In total, 57,053 participants were recruited. Eligible participants were born in Denmark and were living in the greater Copenhagen or Aarhus areas. A FFQ and a lifestyle questionnaire were filled in by each participant. All participants visited a study center, where trained laboratory technicians conducted anthropometric measurements including height, weight and waist circumference, and the self-administered questionnaires were 
Accepted manuscript

scanned and interviewer-checked. A thorough description of the data collection process has been published previously ${ }^{(15)}$. The study was approved by the relevant regional ethical committees on human studies and by the Danish Data Protection Agency. All participants gave written informed consent.

\section{Dietary assessment}

The participants filled in a 192-item semi quantitative FFQ, which was designed for the study ${ }^{(16)}$ and validated against two 7-day weighed diet records ${ }^{(17)}$. Participants reported their average intake of different food and beverage items over the previous year within 1 of 12 possible intake categories that ranged from never to $\geq 8$ times/day. The FFQ included 8 questions specifically about potatoes (boiled (eaten hot for dinner, cold for dinner as potato salad or cold for lunch in an open sandwich), baked, roasted, fried, mashed, or stewed) and 25 questions specifically about other vegetables (e.g. raw, cooked, or baked). Most of the questions covered intake of single vegetable items, like carrots, peas and beans. Few questions covered intake of dishes with vegetables as the main ingredient, like green salad and vegetable steak. In addition to these vegetable-specific questions, minor contributions to the vegetable intake came from dishes like goulash and stew. The daily intake of potatoes and other vegetables was calculated for each participant by using the software program FoodCalc ${ }^{(18)}$. Intake of potatoes was combined into one potato group, with the majority being boiled potatoes. Intake of potato chips was not included in the potato variable. The other vegetables were further grouped based on a botanical definition: fruiting vegetables (cucumber, eggplant, green pepper, red pepper, tomato, zucchini, avocado, green beans, green peas, corn), root vegetables other than potatoes (carrot, celeriac, ginger), and cabbage (white cabbage, broccoli, red cabbage, spring cabbage, Brussels sprouts). The intake of mushrooms, onions, stalk vegetables/sprouts, and leafy vegetables was limited, and these vegetable groups were combined into a group named remaining vegetables. Potatoes, total vegetables other than potatoes, fruiting vegetables, root vegetables (not including potatoes), and cabbage were investigated as exposure groups.

\section{Identification of cases}

The outcome measure was incident non-fatal and fatal MI. Participants registered with a first-time discharge diagnosis of MI (International Classification of Diseases (ICD) 8th revision codes 410-410.99 and ICD 10th revision codes I21.0-I21.9) were identified through linkage with the Danish National Patient Register and the Danish Cause of Death Register using the unique tendigit civil registration number provided to all Danish citizens. Furthermore, we included participants, who were registered with a diagnosis of cardiac arrest (ICD, 8th revision, code 427.27 or ICD, 10th revision, codes I46.0-I46.9) provided that the arrest was believed to be of cardiac 
Accepted manuscript

origin after validation. From baseline through 2003, the medical records were retrieved and reviewed, and the diagnoses were validated in accordance with the criteria of MI as recommended by the American Heart Association and other major health organizations for use in epidemiologic studies ${ }^{(19-21)}$. From January 1, 2004 and until the end of study on December 31, 2009, all participants with a hospital diagnosis of MI were accepted without further validation, as the diagnoses in the Danish National Patient Register has a positive predictive value above $92 \%{ }^{(19)}$. All other potential cases were validated individually by investigating all diagnoses and procedure codes of the involved participants in the National Patient Register and the Cause of Death Register.

\section{Assessment of covariates}

From the lifestyle questionnaire we obtained information on length of education, smoking status, physical activity, menopausal status (women only), and use of postmenopausal hormones (women only) together with history of hypertension, hypercholesterolemia and diabetes mellitus.

Total length of elementary and secondary education was reported in predefined categories $(\leq 7,8-10$ and $>10$ years). Smoking status was reported as never, former, or current and if current smoker the number of cigarettes, cigars, cheroots, and tobacco pipes smoked per day. Current tobacco consumption was calculated by using conversion factors of 1.0 for cigarettes, 4.5 for cigars, and 3.0 for cheroots and tobacco pipes, and divided in $<15,15-25$ or $>25 \mathrm{~g}$ tobacco per day. Physical activity was reported as planned physical activity and active transportation in winter and summer and divided into $<$ or $\geq 3.5 \mathrm{~h}$ per week. History of hypertension was defined as reported hypertension and/or use of antihypertensive medications (yes, no, don't know). History of hypercholesterolemia was defined as reported hypercholesterolemia and/or use of cholesterol lowering medications (yes, no, don't know). History of diabetes mellitus was defined as reported diabetes mellitus and/or use of insulin (yes, no or don't know). BMI was calculated as weight in kilograms divided by height in meters squared $\left(\mathrm{kg} / \mathrm{m}^{2}\right)$ as measured at the visit to the study center. Waist circumference was likewise measured by a trained laboratory technician at the study center. Information on alcohol intake, total energy intake and intake of other food groups was obtained from the FFQ.

\section{Exclusion criteria}

Participants with a diagnosis of cancer at study enrolment that was not registered in the Danish Cancer Registry at the time of invitation (due to processing delay) were excluded. Furthermore, participants registered with a previous diagnosis of MI or cardiac arrest at baseline were excluded. Finally, participants with incomplete questionnaires or missing anthropometric measurements were also excluded. 


\section{Statistical analyses}

The Cox proportional hazards model was used to calculate hazard ratios (HR) with 95\% CI for specified vegetable substitutions and risk of MI. Analyses were carried out separately for women and men. The participants' age was used as the underlying time scale, and the observation time for each participant began at enrollment into the Diet, Cancer and Health cohort (between December 1993 and May 1997) and ended on the date of a registered diagnosis of MI, death from another cause, emigration, or December 31, 2009, whichever occurred first. All models were stratified by age and date of enrolment to allow for differences in baseline hazards by entering these variables into the strata statement of the Cox models.

Vegetable substitutions were investigated in both $\mathrm{g} /$ week and $\mathrm{kcal} /$ week by including the exposure food groups as continuous variables. All substitutions were modelled statistically and thus based on differences in dietary habits at baseline and not on actual food replacements by the study participants. For substitutions between potatoes, other vegetables, fruiting vegetables and root vegetables other than potatoes, increments of $500 \mathrm{~g} /$ week or $250 \mathrm{kcal} /$ week $(=1,046 \mathrm{~kJ} /$ week $)$ were used. For substitution of cabbage for potatoes or other vegetable subgroups, increments of 200 $\mathrm{g} /$ week or $100 \mathrm{kcal} /$ week $(=418.4 \mathrm{~kJ} /$ week) were chosen due to the limited variation in intake of cabbage. The substitutions were calculated by including each of the vegetable subgroups in the statistical models, except for the vegetable group to be replaced, in addition to a sum variable made up by the total vegetable intake (the sum of potatoes, fruiting vegetables, root vegetables other than potatoes, cabbage, and remaining vegetables). For example, the replacement of potatoes with other vegetables (the sum of fruiting vegetables, root vegetables other than potatoes, cabbage, and remaining vegetables) was specified in the statistical models by inclusion of the variable for other vegetables and the sum variable made up by total vegetable intake, thereby leaving out potatoes from the model. Because the total amount of vegetables was held constant by inclusion of the sum variable in the statistical models, $500 \mathrm{~g} /$ week increments of other vegetables implied a concomitant $500 \mathrm{~g} /$ week lower intake of potatoes that were excluded from the model. This method has been named the leave-one-out method and is described in more detail in the method paper on food substitution models for nutritional epidemiology by Ibsen et al. ${ }^{(22)}$. Model 1a included age and date of enrolment as described above and total energy intake (kcal/week). By including total energy intake in the models, we compared participants with similar energy intake but with different composition of the diet. Therefore, the HR for replacement of e.g. potatoes with other vegetables may be interpreted as the estimated difference in risk for a $500 \mathrm{~g} / \mathrm{week}$ or $250 \mathrm{kcal} / \mathrm{week}$ higher intake of other vegetables and a concomitantly lower intake of potatoes for the same level of total energy intake. Model $1 \mathrm{~b}$ included variables in model 1a plus established risk factors for MI: BMI $\left(\mathrm{kg} / \mathrm{m}^{2}\right)$, waist circumference $(\mathrm{cm})$, length of elementary and secondary education $(\leq 7,8-10$, and 
Accepted manuscript

$>10$ years), smoking status (never, former, current smokers of $<15,15-25$ or $>25 \mathrm{~g} / \mathrm{d}$ ), leisure-time physical activity (< or $\geq 3.5 \mathrm{~h} /$ week), alcohol abstinence (yes, no), alcohol consumption (g/d), menopausal status (premenopausal, postmenopausal, unknown) (women only), and use of postmenopausal hormones (never, former, current) (women only). Model 2 included all variables in model $1 \mathrm{~b}$ and potential dietary confounders for the investigated associations: fruits, unprocessed red meat, processed red meat, poultry, lean fish, fatty fish, lean dairy products, fatty dairy products, refined grains, whole grains, nuts, potato chips, sweets, and soft drinks (all g/week). Model 3 included variables in model 2 and possible intermediate factors: history of hypertension (yes, no, don't know), history of hypercholesterolemia (yes, no, don't know) and history of diabetes mellitus (yes, no, don't know). Adjustment for BMI, waist circumference and alcohol consumption was expanded beyond simple linear or categorical approaches to include flexible curves via restricted cubic splines (3 knots) ${ }^{(23)}$. As a sensitivity analysis, we also ran our models without adjustment for total energy intake. We furthermore ran stratified analyses by quintiles of energy adjusted intake of other vegetables. We also tested for interaction by other vegetable intake by including an interaction term between other vegetable intake and each of the two food groups of interest in each substitution analysis. We used likelihood ratio tests to compare models with and without interaction terms in substitution models that included all exposures of interest (these models are equivalent to the models used in our main analyses ${ }^{(22)}$ ). The assumption of proportional hazards in the Cox models was investigated by graphical assessment and was found to be appropriate in all models.

Differences in the underlying contributing dietary patterns were investigated using radar plots of energy-adjusted median intake of food and beverage groups (fruits, unprocessed red meat, processed red meat, poultry, lean fish, fatty fish, lean dairy products, fatty dairy products, refined grains, whole grains, nuts, potato chips, sweets, soft drinks, and alcohol) by energy-adjusted extreme quintiles of intakes of potatoes or other vegetables. Energy-adjusted intake was calculated using the residual method ${ }^{(24)}$. Sex-specific median intake of each food group was used as the common denominator to calculate percentage-wise deviations.

All analyses were performed with use of Stata Statistical Software release 12 and 16 (Stata Corporation, College Station, TX) at a two-tailed alpha of 0.05 .

\section{RESULTS}

Among the 57,053 participants recruited, 567 participants with a cancer diagnosis and 900 participants with a diagnosis of MI or cardiac arrest before baseline were excluded as well as 415 participants with incomplete questionnaires or missing anthropometric measurements. The final study population therefore included 55,171 participants (29,142 women and 26,029 men) with baseline characteristics given in Table 1. During a median follow-up time of 13.6 years for women 
Accepted manuscript

and 13.5 years for men, 656 female and 1,694 male cases with a diagnosis of MI were identified. The median energy-adjusted intakes of potatoes, other vegetables and subgroups of vegetables in $\mathrm{g} /$ week and $\mathrm{kcal} /$ week are shown in Table 2. Intake of potatoes contributed the most to the total vegetable intake and cabbage contributed the least.

Table 3 shows HRs and 95\% CIs for MI associated with replacement of potatoes with other vegetables and substitutions between vegetable subgroups among women. After adjustments for established risk factors of MI, the associations were weak and not statistically significant (model 1b). The HR for MI associated with replacement of $500 \mathrm{~g} / \mathrm{week}$ potatoes with other vegetables was 1.02 (95\% CI: $0.93,1.13)$. The HR for replacement of potatoes with fruiting vegetables was 0.93 (95\% CI: 0.77, 1.13) and with other root vegetables 0.91 (95\% CI: 0.77, 1.07). Direct but statistically non-significant associations were observed for a higher intake of cabbage replacing potatoes, fruiting vegetables or root vegetables. No substantial differences in the observed pattern of associations were found after additional adjustment for other foods (model 2) or after further adjustment for history of hypertension, hypercholesterolemia or diabetes mellitus at baseline (results not shown). Weekly replacements in kcal are presented in Supplemental table S1 and the findings were similar to those in $\mathrm{g} / \mathrm{week}$.

Table 4 shows HRs and 95\% CIs for MI associated with replacement of potatoes with other vegetables and substitutions between vegetable subgroups among men in $\mathrm{g} / \mathrm{week}$. The associations were weak and not statistically significant after adjustment for established risk factors of MI (model 1b). Overall, the pattern of associations was found to be similar to that for women. Weekly replacements in kcal are presented in Supplemental table S2, and the findings were similar to those in $\mathrm{g} /$ week.

The findings from substitution models in $\mathrm{g} /$ week without adjustment for total energy intake were similar to the models with adjustment for total energy intake (results not shown). We did not find indication of effect modification by other vegetable intake (Supplemental tables S3 and S4).

Sex-specific radar plots of median intake of foods and beverages among participants within extreme quintiles of potato intake or extreme quintiles of other vegetable intake are presented in Figure 1 and Figure 2. In general, the eating patterns among women and men were similar. Participants in the highest quintile of potato intake consumed more unprocessed and processed red meat and less fruits, nuts, and lean dairy products compared to participants in the lowest quintile of potato intake. In contrast, participants in the highest quintile of other vegetable intake consumed more fruits, poultry, fish, and nuts and less processed red meat, sweets, and soft drinks compared with participants in the lowest quintile of other vegetable intake. 


\section{DISCUSSION}

In this cohort study including 2,350 cases with a diagnosis of MI, we investigated whether a lower intake of potatoes replaced with a higher intake of other vegetables or replacements between subgroups of other vegetables was associated with the risk of MI. We observed that the associations between substitution of potatoes with other vegetables or vegetable subgroups and risk of MI were weak and statistically non-significant. Likewise, the associations of substitution between subgroups of other vegetables and risk of MI were weak and not statistically significant.

Main strengths of this population-based cohort study are the nearly complete follow-up (99.6\%), the large number of validated cases, the validated assessment of dietary intake, and the detailed information about a wide range of potential confounders. The study population consisted of middle-aged subjects and was slightly overrepresented by participants of higher socioeconomic status. We do, however, expect the direction of the investigated associations to be independent of socioeconomic status. Information bias is unlikely to have affected the results as events of MI were identified by record linkage to the Danish National Patient Register and the Cause of Death Register, and diagnoses were established independently of the dietary information provided by the participants. Due to a low positive predictive value of the diagnosis unstable angina ${ }^{(19)}$, unstable angina was not included in the outcome definition. We used FFQs to assess the dietary intake, and this instrument may reflect the habitual diet ${ }^{(15,16)}$. However, a potential source of random measurement error arises from dietary self-reporting. Also, the use of standard recipes to partly estimate intake of vegetables may be a potential source of random measurement error. The FFQs were completed between 1993 and 1997, and even though we do not expect most people between 50-64 years to change their diet substantially, the food market has changed during follow-up. The dietary habits of the study participants might also have changed, for example due to awareness of the presence of cardiometabolic risk factors, and measurement error due to change in diet during follow-up cannot be excluded. For instance, study participants in high risk of developing MI might have shifted to a higher vegetable intake during follow-up.

Detailed control for established risk factors for MI attenuated the measures of association, and residual confounding cannot be excluded. Confounding from other MI risk factors not taken into account may also contribute to explaining the observed associations. Total energy intake was included as a covariate in all models. This implies that participants with similar total energy intake are compared. We also ran our models without adjustment for total energy intake and found comparable results. A high vegetable intake may be an indicator of a healthier eating pattern and a healthier lifestyle in general ${ }^{(25-27)}$. The healthy eating pattern was confirmed in radar plots of selected foods and food groups among participants in the present study. Participants with the highest intake of vegetables other than potatoes also had the highest intake of fruits, poultry, fish 
and nuts and the lowest intake of processed red meat, sweets and soft drinks compared with participants with the lowest intake of other vegetables. In contrast, we did not observe a similar healthy eating pattern when comparing participants with the highest potato intake with participants with the lowest potato intake. In the substitution models, we therefore adjusted for the underlying eating pattern in additional multivariable models. Although results from models with and without additional adjustment for other foods must be interpreted differently, the pattern of associations were similar. In substitution models not adjusted for other dietary factors (model $1 \mathrm{~b}$ ), the underlying dietary pattern associated with different choices of potatoes or other vegetables is allowed to vary according to potato and other vegetable intake, whereas models adjusted for other dietary factors (model 2) restrict differences in other aspects of the diet composition. In relation to dietary guidelines in a public health perspective, we consider the results from model $1 \mathrm{~b}$ most relevant as these take into account that a given food choice is naturally accompanied by intake of other foods. Among others, hypertension, hypercholesterolemia and diabetes mellitus may be considered both as risk factors for MI and as intermediate factors in the causal pathway between vegetable intake and development of MI. However, a similar pattern of associations was observed after adjustment for these factors.

Several nutrients and bioactive components in vegetables such as dietary fiber, vitamins, minerals, and phytochemicals have been suggested to have a beneficial effect on risk factors of MI, such as hypertension and inflammation ${ }^{(28,29)}$. Potatoes are a source of vitamin C, potassium, magnesium, fiber and polyphenols, but compared to most other vegetables, potatoes are also energy dense and have a high glycemic index, although this may vary with preparation method ${ }^{(14)}$. Hence, a higher intake of other vegetables and a concomitant lower intake of potatoes may act through multiple mechanisms to reduce the risk of MI. However, we did not observe a lower risk of MI associated with substitution of other vegetables for potatoes.

Several cohort studies have examined the association between vegetable intake and risk of CHD while adjusting for total energy intake without specifying the replacement food. For total vegetables, the combined evidence from prospective studies shows an inverse association with CHD, although findings from the individual studies are heterogeneous ${ }^{(5,6)}$. For subgroups of vegetables, two recent meta-analyses have summarized the evidence for potatoes and found no association between potato consumption and CHD ${ }^{(6,12)}$. For other vegetable subgroups, no associations or inverse associations, especially for green leafy vegetables, were reported ${ }^{(6)}$. The heterogeneity between studies may be due to differences in the methodological approaches. As individuals usually change the dietary composition to maintain energy balance, a higher vegetable intake might replace other energy-providing foods in the diet which may have beneficial, neutral or detrimental effects with consequences for the interpretation of the study results. In the present study, substitutions between vegetable subgroups were not associated with risk of MI. However, the 
effects of substitution of vegetables may vary depending on the categorization of the vegetables. We used a botanical categorization but other classifications may be relevant to CHD risk such as the fiber content and glycemic index of the vegetables. Substitutions were investigated in $\mathrm{g} /$ week and $\mathrm{kcal} /$ week. An advantage of investigating substitutions in $\mathrm{g} / \mathrm{week}$ is the straightforward translation into food-based dietary guidelines, while investigation of substitutions in kcal/week is not easily translated. Substitution in kcal/week, however, has an isoenergetic interpretation whereas substitutions in $\mathrm{g} /$ week may introduce an unspecified substitution of energy from other foods. The energy content of 200 grams of e.g. cooked broccoli is $70 \mathrm{kcal}$, and the energy content of 200 grams of e.g. boiled potato is $170 \mathrm{kcal}^{(30)}$. Thus, substituting the same absolute amount of cooked broccoli for potatoes is also followed by a higher intake of other foods. In the present study, however, the pattern of associations for $\mathrm{g} / \mathrm{week}$ was similar to that for $\mathrm{kcal} / \mathrm{week}$.

In conclusion, this study found no benefits of replacing potatoes with other vegetables and no benefits of replacements between vegetable subgroups for prevention of MI. Thus, the study supports food-based dietary guidelines recommending to consume a variety of vegetables from all subgroups.

Acknowledgements

We thank the staff from the Danish Cancer Society who kindly provided the data necessary for our analysis. The Danish Diet, Cancer and Health cohort was approved by the regional ethical committees of human studies in Copenhagen and Aarhus and by the Danish Data Protection Agency.

\section{Financial Support}

This work is part of the project 'Diet and prevention of ischemic heart disease: a translational approach' (DIPI, www.dipi.dk), which was supported by the Danish Council for Strategic Research (today, Innovation Fund Denmark) (Contract 0603-00488B). This work was furthermore supported by the Graduate School of Health at Aarhus University and the Danish Cancer Society. The funding agencies had no role in the design, analysis or writing of this article.

\section{Conflict of interest}

None

\section{Authorship}

The contributions of the authors were as follows: A. M. L. W., M. D. H., K. O. and M. U. J. designed the study; A. T. and K. O. collected the data; A. M. L. W. and M. D. H. were the principal investigators and performed the data analyses. A. M. L. W. and M. D. H. wrote the manuscript. A. 
Accepted manuscript

T., E. B. S., E. B. R., K. O. and M. U. J. contributed to the data analysis and to the writing of the manuscript. All authors have read and approved the final manuscript.

Supplementary Material: Supplemental table S1, Supplemental table S2, Supplemental table S3 and Supplemental table S4. 


\section{REFERENCES}

1. Wilkins E, Wilson L, Wickramasinghe K, Bhatnagar P, Leal J, Luengo-Fernandez R, Burns R, Rayner M, Townsend N (2017) European Cardiovascular Disease Statistics 2017. European Heart Network, Brussels. Available at: http://www.ehnheart.org/cvdstatistics/cvd-statistics-2017.html.

2. Benjamin EJ, Muntner P, Alonso A et al. (2019) Heart Disease and Stroke Statistics-2019 Update: A Report From the American Heart Association. Circulation 139, e56-e528.

3. Hu FB \& Willett WC (2002) Optimal diets for prevention of coronary heart disease. JAMA : the journal of the American Medical Association 288, 2569-2578.

4. Dalen JE \& Devries S (2014) Diets to prevent coronary heart disease 1957-2013: what have we learned? The American Journal of Medicine 127, 364-369.

5. Bechthold A, Boeing H, Schwedhelm C et al. (2019) Food groups and risk of coronary heart disease, stroke and heart failure: A systematic review and dose-response meta-analysis of prospective studies. Crit Rev Food Sci Nutr 59, 1071-1090.

6. Aune D, Giovannucci E, Boffetta P et al. (2017) Fruit and vegetable intake and the risk of cardiovascular disease, total cancer and all-cause mortality-a systematic review and doseresponse meta-analysis of prospective studies. Int J Epidemiol 46, 1029-1056.

7. Lichtenstein AH, Appel LJ, Brands M et al. (2006) Diet and lifestyle recommendations revision 2006: a scientific statement from the American Heart Association Nutrition Committee. Circulation 114, 82-96.

8. European Heart Network (2017). Transforming European food and drink policies for cardiovascular health. Brussels. Available at: http://www.ehnheart.org/publications-andpapers/publications/1093:transforming-european-food-and-drinks-policies-forcardiovascular-health.html

9. Hall KD \& Guo J (2017) Obesity Energetics: Body Weight Regulation and the Effects of Diet Composition. Gastroenterology 152, 1718-1727.e1713.

10. U.S. Department of Health and Human Services and U.S. Department of Agriculture. 2015 2020 Dietary Guidelines for Americans. 8th Edition. December 2015. Available at https://health.gov/dietaryguidelines/2015/guidelines/. 
11. Willett WC (2017) Will it be cheese, bologna, or peanut butter? Eur J Epidemiol 32, 257259.

12. Schwingshackl L, Schwedhelm C, Hoffmann G et al. (2019) Potatoes and risk of chronic disease: a systematic review and dose-response meta-analysis. Eur J Nutr 58, 2243-2251.

13. Bertoia ML, Mukamal KJ, Cahill LE et al. (2015) Changes in Intake of Fruits and Vegetables and Weight Change in United States Men and Women Followed for Up to 24 Years: Analysis from Three Prospective Cohort Studies. PLoS medicine 12, e1001878.

14. Furrer AN, Chegeni M Ferruzzi MG (2018) Impact of potato processing on nutrients, phytochemicals, and human health. Crit Rev Food Sci Nutr 58, 146-168.

15. Tjonneland A, Olsen A, Boll K et al. (2007) Study design, exposure variables, and socioeconomic determinants of participation in Diet, Cancer and Health: a population-based prospective cohort study of 57,053 men and women in Denmark. Scandinavian Journal of Public Health 35, 432-441.

16. Overvad K, Tjonneland A, Haraldsdottir J et al. (1991) Development of a semiquantitative food frequency questionnaire to assess food, energy and nutrient intake in Denmark. International journal of epidemiology 20, 900-905.

17. Tjonneland A, Overvad K, Haraldsdottir J et al. (1991) Validation of a semiquantitative food frequency questionnaire developed in Denmark. International journal of epidemiology 20, 906-912.

18. FoodCalc. Danish Cancer Society. Available at https://www.cancer.dk/dchdata/access-todata-and-biobank/foodcalc/ (accessed Mar 4 2020)

19. Joensen AM, Jensen MK, Overvad K et al. (2009) Predictive values of acute coronary syndrome discharge diagnoses differed in the Danish National Patient Registry. Journal of clinical epidemiology 62, 188-194.

20. Luepker RV, Apple FS, Christenson RH et al. (2003) Case definitions for acute coronary heart disease in epidemiology and clinical research studies: a statement from the AHA Council on Epidemiology and Prevention; AHA Statistics Committee; World Heart Federation Council on Epidemiology and Prevention; the European Society of Cardiology Working Group on Epidemiology and Prevention; Centers for Disease Control and Prevention; and the National Heart, Lung, and Blood Institute. Circulation 108, 2543-2549. 
21. Stegger JG, Schmidt EB, Obel T et al. (2011) Body composition and body fat distribution in relation to later risk of acute myocardial infarction: a Danish follow-up study. Int J Obes (Lond) 35, 1433-1441.

22. Ibsen DB, Laursen ASD, Würtz AML et al. (2020) Food substitution models for nutritional epidemiology. Am J Clin Nutr.

23. Greenland S (1995) Dose-response and trend analysis in epidemiology: alternatives to categorical analysis. Epidemiology 6, 356-365.

24. Willett WC (2013) Implications of total energy intake for epidemiologic analyses. In Nutritional Epidemiology, 3rd ed., pp. 260. USA: Oxford University Press.

25. Thompson RL, Margetts BM, Speller VM et al. (1999) The Health Education Authority's health and lifestyle survey 1993: who are the low fruit and vegetable consumers? $J$ Epidemiol Community Health 53, 294-299.

26. Meader N, King K, Moe-Byrne T et al. (2016) A systematic review on the clustering and co-occurrence of multiple risk behaviours. BMC Public Health 16, 657.

27. Imamura F, Lichtenstein AH, Dallal GE et al. (2009) Confounding by dietary patterns of the inverse association between alcohol consumption and type 2 diabetes risk. Am J Epidemiol $170,37-45$.

28. Alissa EM \& Ferns GA (2017) Dietary fruits and vegetables and cardiovascular diseases risk. Crit Rev Food Sci Nutr 57, 1950-1962.

29. Boeing H, Bechthold A, Bub A et al. (2012) Critical review: vegetables and fruit in the prevention of chronic diseases. European journal of nutrition 51, 637-663.

30. Fødevaredata (Fooddata, www.frida.fooddata.dk), version 4, The National Food Institute, Technical University of Denmark. Accessed March 4, 2020. 
Accepted manuscript

\section{TABLES AND FIGURES}

\section{Table 1}

Baseline characteristics of the study population in the Diet, Cancer and Health cohort

\begin{tabular}{|c|c|c|}
\hline Characteristics & $\begin{array}{c}\text { Women } \\
(n=29,142)\end{array}$ & $\begin{array}{c}\text { Men } \\
(n=26,029)\end{array}$ \\
\hline Age (y) & $56(52-60)$ & $55(52-60)$ \\
\hline $\mathrm{BMI}\left(\mathrm{kg} / \mathrm{m}^{2}\right)$ & $25(23-28)$ & $26(24-29)$ \\
\hline Waist circumference $(\mathrm{cm})$ & $80(74-88)$ & $95(89-102)$ \\
\hline \multicolumn{3}{|l|}{ Education, \% (n) } \\
\hline$<7 \mathrm{y}$ & $31(9,107)$ & $34(8,920)$ \\
\hline $8-10 y$ & $50(14,630)$ & $42(10,856)$ \\
\hline$>10 \mathrm{y}$ & $19(5,405)$ & $24(6,253)$ \\
\hline \multicolumn{3}{|l|}{ Smoking status, \% (n) } \\
\hline Never & $44(12,759)$ & $26(6,787)$ \\
\hline Former & $23(6,822)$ & $34(8,910)$ \\
\hline Current $(<15 \mathrm{~g}$ tobacco/d $)$ & $15(4,469)$ & $11(2,765)$ \\
\hline Current $(15-25 \mathrm{~g}$ tobacco/d) & $15(4,336)$ & $18(4,554)$ \\
\hline Current $(>25 \mathrm{~g}$ tobacco/d) & $3(756)$ & $12(3,013)$ \\
\hline \multicolumn{3}{|l|}{ Leisure-time physical activity, \% (n) } \\
\hline$<3.5 \mathrm{~h}$ per week & $59(17,272)$ & $62(16,183)$ \\
\hline$\geq 3.5 \mathrm{~h}$ per week & $41(11,870)$ & $38(9,846)$ \\
\hline Abstainers, \% (n) & $3(797)$ & $2(473)$ \\
\hline Alcohol intake $(\mathrm{g} / \mathrm{d})^{*}$ & $10(3-18)$ & $20(11-41)$ \\
\hline Total energy intake (kcal/d) & $1,922(1,604-2,290)$ & $2,364(1,996-2,793)$ \\
\hline Postmenopausal status, $\%$ (n) & $59(17,109)$ & - \\
\hline Current menopausal hormone use, \% (n) & $30(8,755)$ & - \\
\hline History of hypertension at baseline, \% (n) & $17(5,012)$ & $15(3,774)$ \\
\hline History of high cholesterol at baseline, $\%$ (n) & $6(1,771)$ & $8(2,012)$ \\
\hline History of diabetes mellitus at baseline, \% (n) & $2(438)$ & $3(682)$ \\
\hline
\end{tabular}

Median and interquartile range in parentheses or proportion and number in parentheses.

*Among users. 
Accepted manuscript

\section{Table 2}

Energy-adjusted intakes of potatoes and other vegetables in $\mathrm{g} / \mathrm{week}$ and kcal/week among women and men in the Diet, Cancer and Health cohort

\begin{tabular}{lcc}
\hline & $\begin{array}{c}\text { Women } \\
(\mathrm{n}=29,142)\end{array}$ & $\begin{array}{c}\text { Men } \\
(\mathrm{n}=26,029)\end{array}$ \\
\hline Grams/week & $773(542-1,069)$ & $1,058(735-1,489)$ \\
Potatoes & $1,220(841-1,690)$ & $1,085(728-1,505)$ \\
Other vegetables & $457(292-666)$ & $410(264-618)$ \\
Fruiting vegetables & $209(108-417)$ & $119(65-237)$ \\
Root vegetables & $112(59-174)$ & $100(52-168)$ \\
Cabbage & $336(231-470)$ & $352(244-492)$ \\
Remaining vegetables & & \\
Kcal/week & $710(501-974)$ & $964(675-1,352)$ \\
Potatoes & $532(369-731)$ & $482(325-667)$ \\
Other vegetables & $200(128-289)$ & $181(112-267)$ \\
Fruiting vegetables & $88(46-177)$ & $51(28-100)$ \\
Root vegetables & $49(26-77)$ & $44(23-75)$ \\
Cabbage & $145(100-208)$ & $158(110-228)$ \\
Remaining vegetables & & \\
\hline
\end{tabular}

Median intake and interquartile range. 
Accepted manuscript

\section{Table 3}

Hazard ratios (HR) and 95\% CI for myocardial infarction associated with substitutions between vegetable groups among women in the Diet, Cancer and Health cohort $(n=29,142)$

\begin{tabular}{|c|c|c|c|}
\hline & HR $(95 \%$ CI $)$ & HR $(95 \%$ CI $)$ & HR $(95 \%$ CI $)$ \\
\hline & Model 1a* & Model $1 b^{\dagger}$ & Model 2 \\
\hline & \multicolumn{3}{|c|}{$500 \mathrm{~g} /$ week } \\
\hline $\begin{array}{l}\text { Other vegetables } \\
\text { for potatoes }\end{array}$ & $0.89(0.81,0.99)$ & $1.02(0.93,1.13)$ & $1.04(0.94,1.16)$ \\
\hline $\begin{array}{l}\text { Fruiting vegetables } \\
\text { for potatoes }\end{array}$ & $0.80(0.65,0.97)$ & $0.93(0.77,1.13)$ & $0.97(0.79,1.18)$ \\
\hline $\begin{array}{l}\text { Root vegetables } \\
\text { for potatoes }\end{array}$ & $0.80(0.67,0.95)$ & $0.91(0.77,1.07)$ & $0.93(0.79,1.11)$ \\
\hline \multirow[t]{2}{*}{$\begin{array}{l}\text { Fruiting for root } \\
\text { vegetables }\end{array}$} & $1.00(0.77,1.29)$ & $1.03(0.80,1.31)$ & $1.04(0.81,1.33)$ \\
\hline & \multicolumn{3}{|c|}{200 g/week } \\
\hline $\begin{array}{l}\text { Cabbage for } \\
\text { potatoes }\end{array}$ & $1.02(0.84,1.23)$ & $1.09(0.91,1.30)$ & $1.10(0.92,1.31)$ \\
\hline $\begin{array}{l}\text { Cabbage for } \\
\text { fruiting vegetables }\end{array}$ & $1.11(0.90,1.38)$ & $1.12(0.91,1.37)$ & $1.11(0.91,1.35)$ \\
\hline $\begin{array}{l}\text { Cabbage for root } \\
\text { vegetables }\end{array}$ & $1.11(0.90,1.37)$ & $1.13(0.92,1.38)$ & $1.13(0.92,1.37)$ \\
\hline
\end{tabular}

\footnotetext{
* Model 1a was stratified by age and date of enrolment and further adjusted for age and total energy intake (kcal). To explore the vegetable substitutions we used the leave-one-out method (please see the statistical methods section for more details). For example, the substitution of other vegetables (fruiting vegetables, root vegetables, cabbage, and remaining vegetables) for potatoes was specified in the statistical models by inclusion of other vegetables and the total sum of potatoes and other vegetables.
}

${ }^{\dagger}$ Model 1b: as model 1a plus BMI, waist circumference, length of education, smoking status, physical activity, alcohol abstinence, alcohol intake, menopausal status, and use of postmenopausal hormones.

${ }^{*}$ Model 2: as model $1 \mathrm{~b}$ plus fruits, unprocessed red meat, processed red meat, poultry, lean fish, fatty fish, lean dairy products, fatty dairy products, refined grains, whole grains, nuts, potato chips, sweets, and soft drinks. 


\section{Table 4}

Hazard ratios (HR) and 95\% CI for myocardial infarction associated with substitutions between vegetable groups among men in the Diet, Cancer and Health cohort $(n=26,029)$

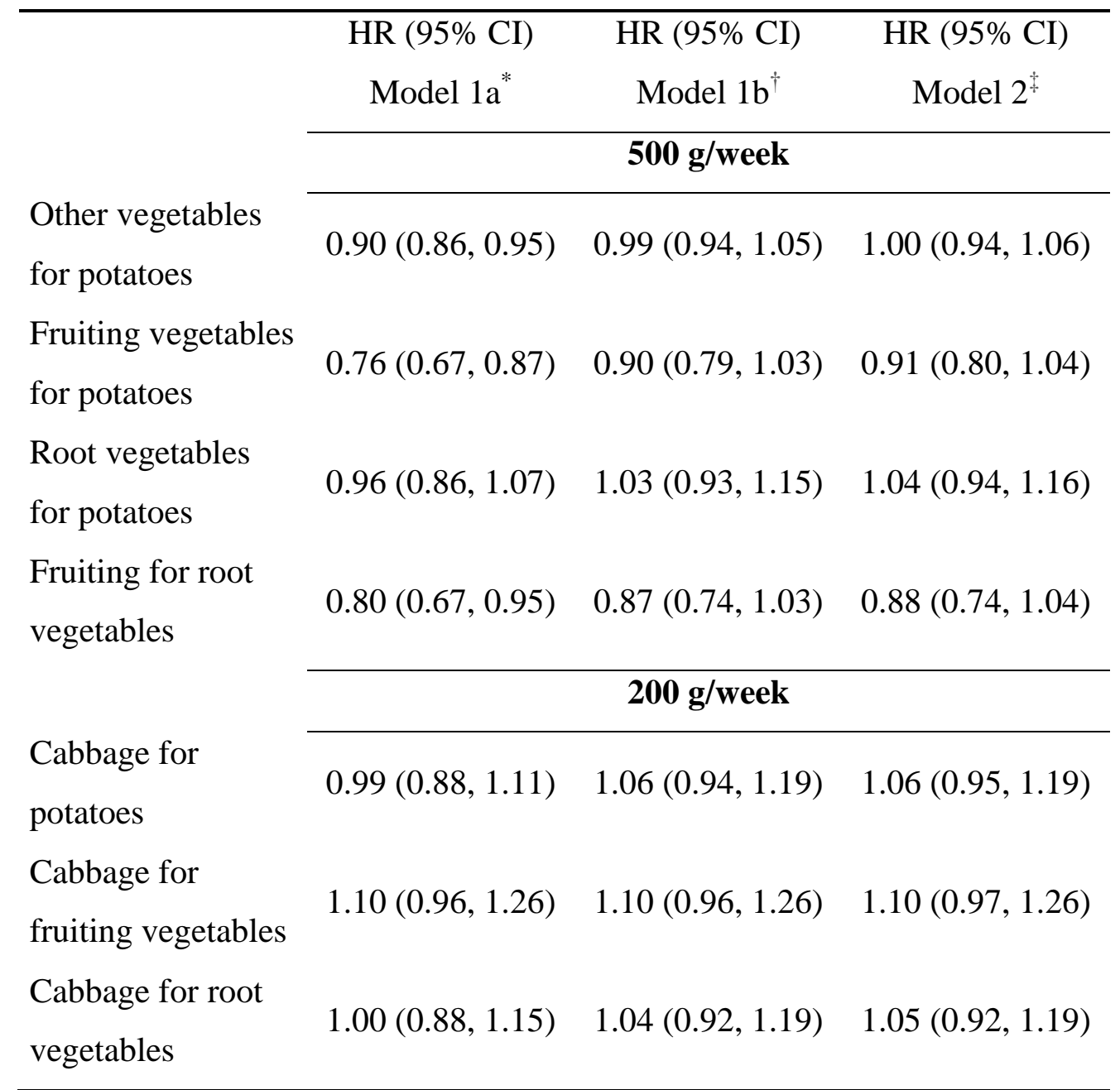

* Model 1a was stratified by age and date of enrolment and further adjusted for age and total energy intake (kcal). To explore the vegetable substitutions we used the leave-one-out method (please see the statistical methods section for more details). For example, the substitution of other vegetables (fruiting vegetables, root vegetables, cabbage, and remaining vegetables) for potatoes was specified in the statistical models by inclusion of other vegetables and the total sum of potatoes and other vegetables.

${ }^{\dagger}$ Model 1b: as model 1a plus BMI, waist circumference, length of education, smoking status, physical activity, alcohol abstinence, and alcohol intake.

${ }^{\ddagger}$ Model 2: as model $1 \mathrm{~b}$ plus fruits, unprocessed red meat, processed red meat, poultry, lean fish, fatty fish, lean dairy products, fatty dairy products, refined grains, whole grains, nuts, potato chips, sweets, and soft drinks. 


\section{FIGURE LEGENDS}
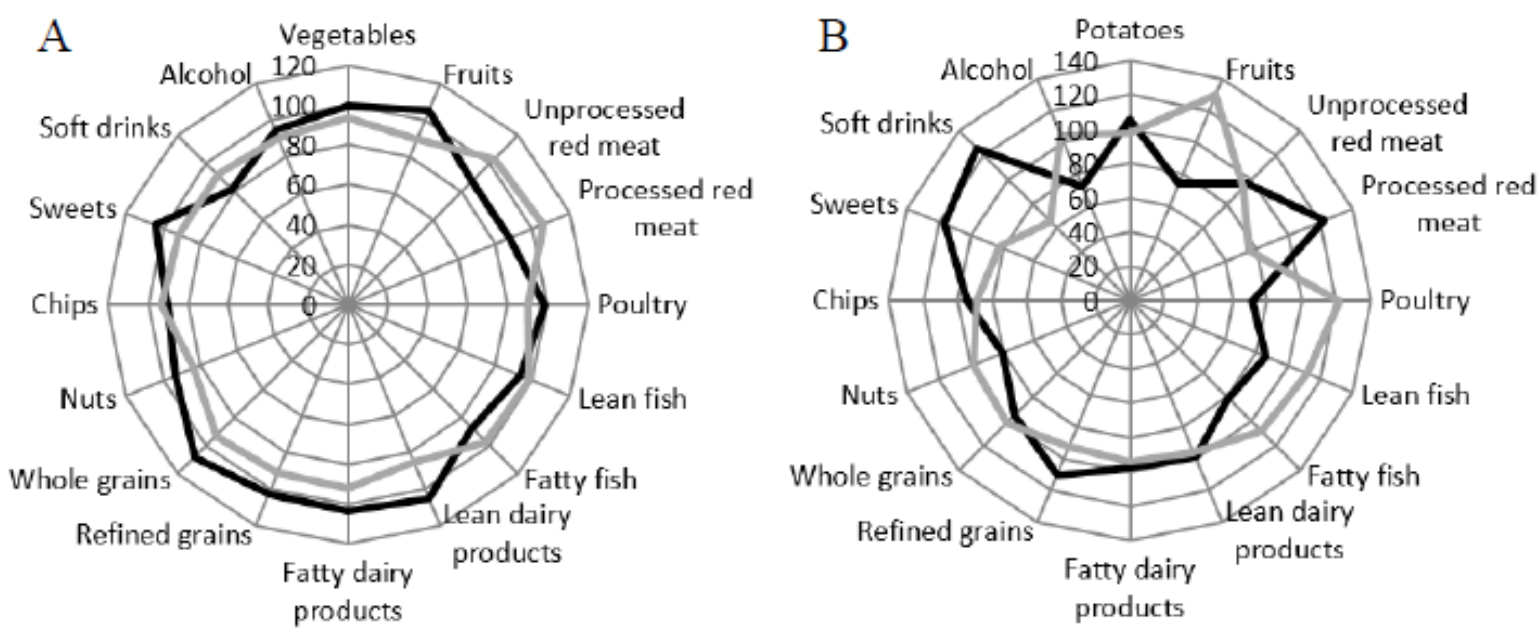

Figure 1. Radar plots illustrating percentage-wise differences in intake of fifteen food and beverage groups by extreme quintiles of potato (panel A) or other vegetable (panel B) intake among women in the Diet, Cancer and Health cohort. Median intake of each food group was used as the common denominator to calculate deviations. If the relative intake of a food is above $100 \%$, it indicates a higher intake of that food compared to the median intake and vice versa when the relative intake is below $100 \%$. Intake of foods was adjusted for total energy intake. The black line indicates the first quintile of potato or other vegetable intake. The grey line indicates the fifth quintile of potato or other vegetable intake. 

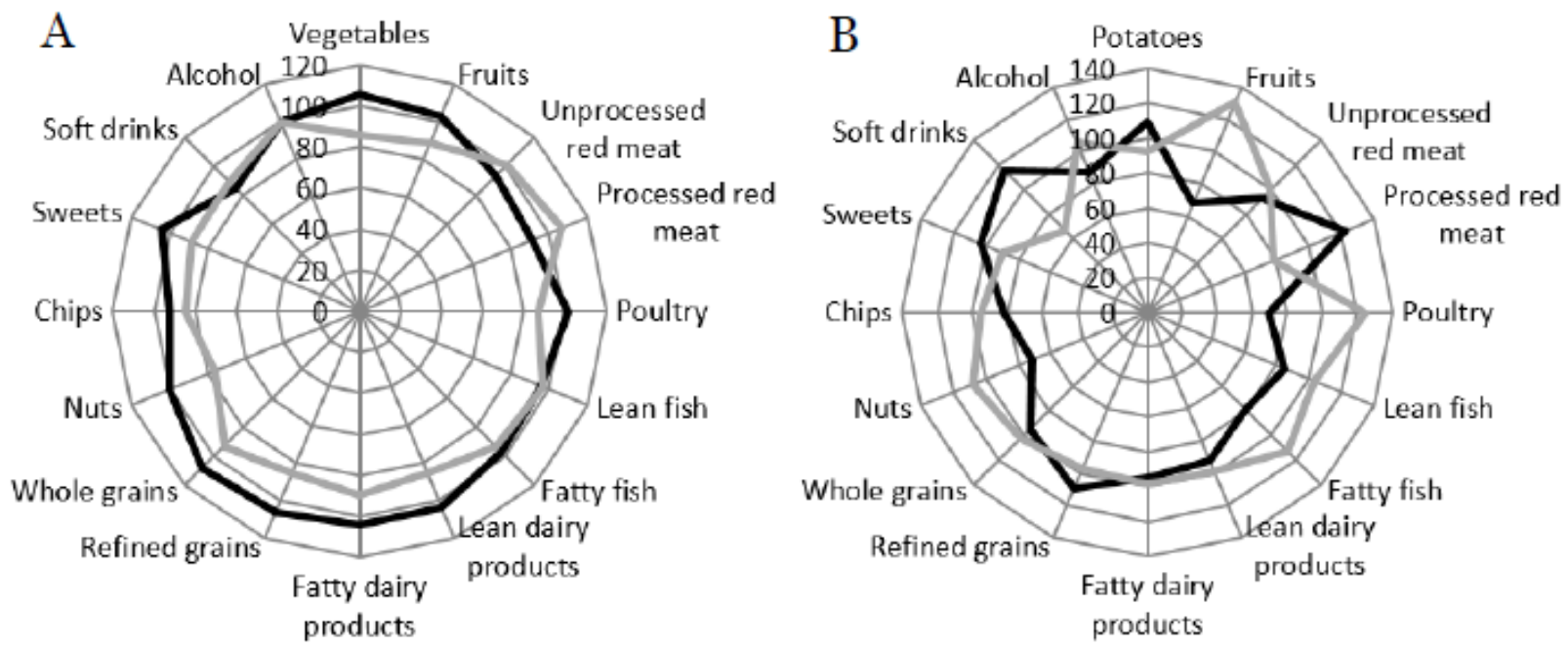

Figure 2. Radar plots illustrating percentage-wise differences in intake of fifteen food and beverage groups by extreme quintiles of potato (panel A) or other vegetable (panel B) intake among men in the Diet, Cancer and Health cohort. Median intake of each food group was used as the common denominator to calculate deviations. If the relative intake of a food is above $100 \%$, it indicates a higher intake of that food compared to the median intake and vice versa when the relative intake is below $100 \%$. Intake of foods was adjusted for total energy intake. The black line indicates the first quintile of potato or other vegetable intake. The grey line indicates the fifth quintile of potato or other vegetable intake. 